\title{
Origin of the double shifted hysteresis loops in thin ferromagnetic films with bias fields
}

\author{
Jyh-Shinn Yang ${ }^{\mathrm{a}, *}$, Ching-Ray Chang ${ }^{\mathrm{b}}$, C.H. Lai ${ }^{\mathrm{c}}$ \\ ${ }^{a}$ Institute of Optoelectronic Sciences, National Taiwan Ocean University, Keelung, Taiwan \\ ${ }^{\mathrm{b}}$ Department of Physics, National Taiwan University, Taipei, Taiwan \\ ${ }^{\mathrm{c}}$ Department of Materials Science and Engineering, Nat'l Tsing Hua University, HsinChu, Taiwan
}

\begin{abstract}
The magnetic switching behavior of thin ferromagnetic films with bias fields is studied by coherent rotation model. The film is taken to be a single magnetic domain with in-plane cubic and uniaxial anisotropies. The origin of occurrence of the double shifted hysteresis loops (DSHLs) is explained in detail. It was shown that the bias field significantly changes the behavior of magnetization process, and the range of material constants for occurrence of DSHLs for biased films is wider than that for unbiased films. (C) 2002 Elsevier Science B.V. All rights reserved.
\end{abstract}

Keywords: Thin films; Coherent rotation; Bias field

The magnetic properties of thin ferromagnetic films have been the subject of intense investigation due to both its great technological importance and fundamental physics. The thin films have unusual properties, resulting from the surface atoms with reduced symmetry, or the change of crystal structure and the morphology of the film [1]. Magnetic anisotropy plays a crucial role in governing the magnetization direction and controlling the magnetic switching process in ultrathin magnetic films. Thus, the determination of anisotropy constant is an essential task in order to understand the magnetic behavior of ultrathin films. There are various well-established methods to determine the magnetic anisotropies, but the novel and convenient approach is to utilize the characteristic shape of double shifted hysteresis loops (DSHLs) [2]. The DSHL comprises two shifted single loops with two distinct nucleation fields, and can be observed along the hard direction in ultrathin magnetic films by applying a constant external field along the easy direction. This gives us a fast and accurate method to determine the magnetic anisotropy in a variety of magnetic systems, by measuring the displacement of the single shifted loop

\footnotetext{
*Corresponding author. Tel.: + 886-2-24622192 ext. 6706; fax: $+886-2-2463-4360$.

E-mail address: jyang@mail.ntou.edu.tw (J.-S. Yang).
}

from the origin and the slope of the loop around zero field [2]. However, the relevant formulae for anisotropy given are empirical and not rigorously derived to date. The micromagnetic analysis has predicted that the DSHLs appear in uniaxial ferromagnets with appropriate ratio of the first- and second-order anisotropy constants [3]. Recently, these loops were observed in biaxial films with an extra uniaxial anisotropy along one of biaxes [4,5], and also in exchange-biased systems [6,7]. Thus, the origin of occurrence of DSHLs in unbiased and biased films should be studied in detail. Moreover, to accurately determine the magnetic anisotropy from hysteresis loops, it is deserved to more fully understand the feature of DSHLs. In this article, we utilize the micromagnetic theory to study the magnetic reversal behavior of ultrathin magnetic films under the existence of a bias field.

In the framework of the coherent rotation model, the thin ferromagnetic film is taken to be a single domain. Noting that the shape anisotropy keeps the magnetization in the plane of the film, the magnetic free energy density of an arbitrary magnetization orientation in the presence of external applied field $H$ and bias field $H_{\mathrm{b}}$ is generally given by

$$
\begin{aligned}
E= & K_{1} \sin ^{2} \theta+K_{2} \sin ^{4} \theta-M_{\mathrm{s}} H \cos (\beta-\theta) \\
& -M_{\mathrm{s}} H_{\mathrm{b}} \cos \theta,
\end{aligned}
$$



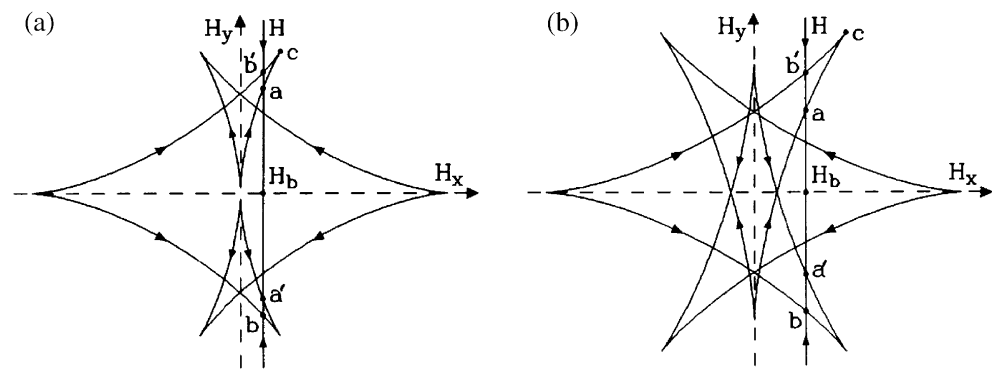

Fig. 1. The oriented critical curves: (a) $-1 / 2<K_{2} / K_{1}<-1 / 6$; (b) $K_{2} / K_{1}<-1 / 2$. The arrowheads in the critical curve indicate the orientation of the tangent to the critical curve. The external magnetic field is applied perpendicular to the easy axis, in combination with the bias field. Here $H_{x}=H \cos \beta$ and $H_{y}=H \sin \beta$.

where $K_{1}$ and $K_{2}$ are the first- and second-order anisotropy constants, including both the contributions from in-plane uniaxial and cubic anisotropies. $M_{\mathrm{s}}$ is the saturation magnetization, $\theta$ the magnetization orientation, and $\beta$ the applied field orientation. $\theta$ and $\beta$ are defined as being zero along the uniaxial easy axis, which is taken here to lie along a cubic easy axis. Namely, $K_{1}=K_{\mathrm{u} 1}+K_{\mathrm{c}}>0$ and $K_{2}=-K_{\mathrm{c}}+K_{\mathrm{u} 2}$, where $K_{\mathrm{c}}$ is the in-plane cubic anisotropy constant, and $K_{\mathrm{u} 1}\left(K_{\mathrm{u} 2}\right)$ is the first (second)-order in-plane uniaxial anisotropy constant. The bias field is assumed to lie along the easy axis for the sake of simplicity. To find the stable direction of $M$, one has to look for the solution of $\mathrm{d} E / \mathrm{d} \theta=0$ and require $\mathrm{d}^{2} E / \mathrm{d} \theta^{2}>0$. To increase our physical understanding of magnetic switching process, it is instructive to establish the locus of critical fields in the field space, the critical curves, which is obtained from the simultaneous consideration of the conditions $\mathrm{d} E / \mathrm{d} \theta=$ $\mathrm{d}^{2} E / \mathrm{d} \theta^{2}=0$.

The characteristic critical curves of present model system are shown in Fig. 1 . As $-1 / 2<K_{2} / K_{1}<-1 / 6$, the customary cusps turn into swallowtails along hard axis; this effect represent a spontaneous orientation transition of magnetization. As the ratio of $K_{2} / K_{1}$ becomes more negative, the critical curve becomes starlike. For unbiased films, the DSHLs only appear around the hard direction of critical curves with swallowtails (Fig. 2). However, the range of material constants for the occurrence of DSHLs can be broadened if a bias field is suitably introduced.

Typically, the bias field points along the easy axis and the applied field sweeps along the hard axis (Fig. 1). As the resultant field line is located within the range of the swallowtail, the stable direction of magnetization is close to the hard axis until the field reach point a at the boundary. As the applied field decreases, the magnetization will jump irreversibly to its equilibrium direction close to the easy axis, and then rotates reversibly until the field reaches point $b$ at the boundary. If the field decreases even further, to exceed the point $b$, the magnetization jumps to an angle of $\theta$ close to $-\pi / 2$.

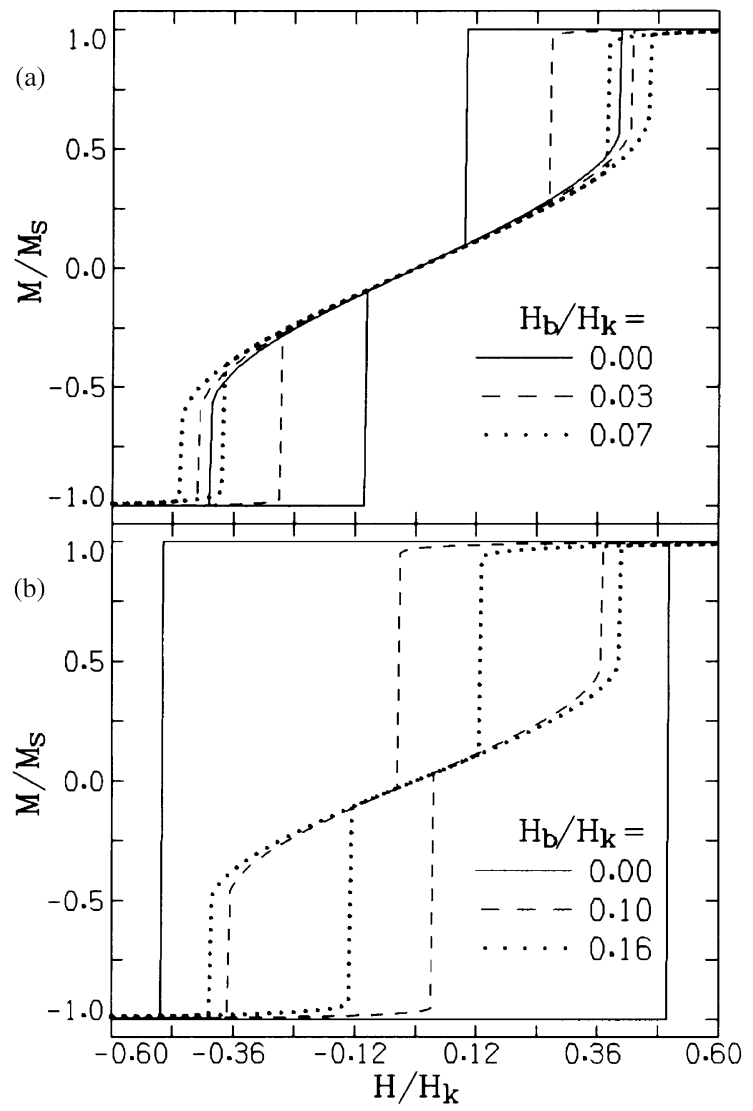

Fig. 2. Calculated hysteresis loops corresponding to the critical curves of Fig. 1. Here $H_{k}=2 K_{1} / M_{\mathrm{s}}$ and $K_{2} / K_{1}=-0.45$ (a) or -0.75 (b).

Apparently, the DSHLs are formed as shown in Fig. 2. To obtain a complete DSHL, we further require the point a to lie above the $H_{x}$ axis and left of point $\mathrm{c}$. The range of the required bias field leading to the occurrence of the DSHLs depends on the ratio of $K_{2} / K_{1}$ (Fig. 3). The influence of bias field on the feature of DSHLs is shown in Fig. 4. Obviously, the shift field $H_{\mathrm{s}}$, defined as 


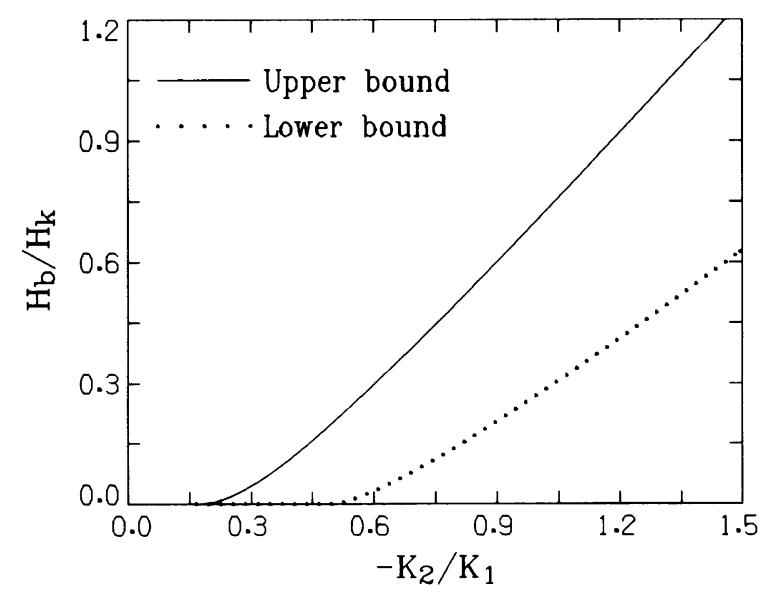

Fig. 3. The upper and lower bounds of the required bias field leading to a complete double shifted loop. Here $H_{k}=2 K_{1} / M_{\mathrm{s}}$.

the field difference between zero field and the center of minor loop, increases monotonously with increasing bias field, but the width of minor loop, $\Delta H$, decreases gradually and reaches to zero finally. It is evident that the variation of shift field with the bias field is generally not linear, in contrast to the commonly cited empirical formula of the shift field [2].

In summary, we have studied the switching behavior of biased thin magnetic films for various ratios of $K_{2} / K_{1}$. Coherent rotation analysis showed that the behavior of magnetic switching critically depends on the ratio of $K_{2} / K_{1}$ and the magnitude of bias field. By suitably introducing the bias field, the complete DSHL can be observed for the regime of $K_{2} / K_{1}<-1 / 6$.

This work was supported by the National Science Council of ROC under Grant No. NSC89-2112-M019-008.

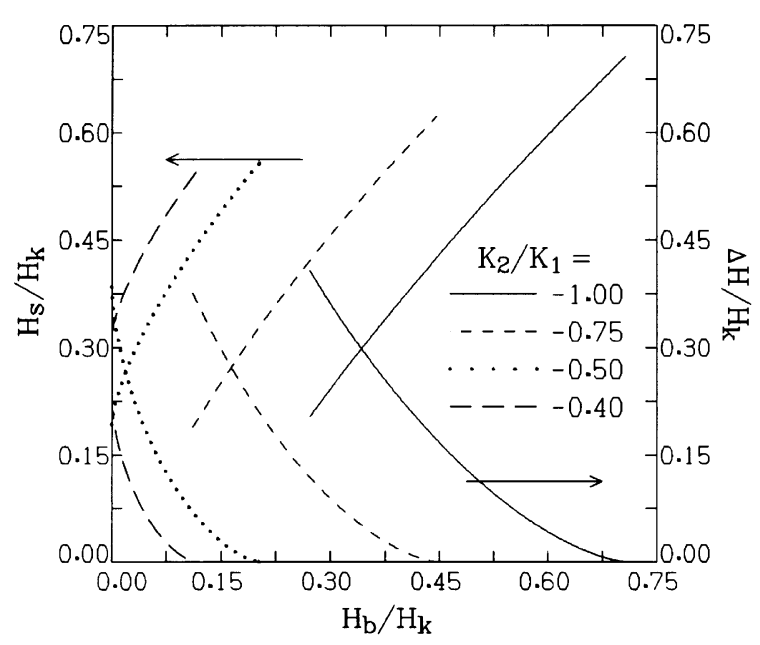

Fig. 4. The dependence of the shift field $H_{\mathrm{s}}$ and width of minor loop $\Delta H$ of complete double shifted loops on bias field for several ratios of $K_{2} / K_{1}$. Here $H_{k}=2 K_{1} / M_{\mathrm{s}}$.

\section{References}

[1] J.A.C. Bland, S. Hope, B. Choi, P. Bode, J. Appl. Phys. 85 (1999) 4613.

[2] W. Weber, R. Allenspach, A. Bischof, Appl. Phys. Lett. 70 (1997) 520.

[3] C.-R. Chang, J. Appl. Phys. 69 (1991) 2431.

[4] C.J. Gutierrez, J.J. Krebs, G.A. Prinz, Appl. Phys. Lett. 61 (1992) 2476.

[5] R.K. Kawakami, E.J. Escorcia-Aparicio, Z.Q. Qiu, Phys. Rev. Lett. 77 (1996) 2570.

[6] Y.J. Tang, X. Zhou, X. Chen, B.Q. Liang, W.S. Zhan, J. Appl. Phys. 88 (2000) 2054.

[7] C.-R. Chang, J.-S. Yang, J.C.A. Huang, C.H. Lai, J. Phys. Chem. Solids 62 (2001) 1737. 